\title{
Joys and Sorrows of Korean Women Undergoing Infertility Treatment: An Ethnographic Perspective
}

\section{Seong-Heui Kim}

Section: Healthcare

Sci. Journal Impact

Factor: 6.1 (2018)

ICV: 90.90 (2018)

Department of Nursing, Daejin University, South Korea.

(c) (7) (8)

Copyright@IJCRR

\section{ABSTRACT}

Introduction: The number of infertile couples are rapidly increasing in Korean society for a variety of reasons, including increased marriage age, stress, environmental pollution, number of working couples, and increased ' 3 abandonments' tendency

Aim and Objective: This study was motivated by the need to develop an effective nursing intervention for women undergoing infertility treatment under Korean sociocultural background. Using ethnographic research method, this study has nine married women who are diagnosed infertile and pursuing medical treatment as informants.

Result: The study has found a common cultural experience from the women undergoing infertility treatment expressed as 'getting rid of stigma of deficient women'. This cultural theme can be divided into the following 4 categories: 'turning into life of a loser', 'doing homework to be recognized for my existence', 'the ambivalence about infertility treatment', 'positioning the value of being myself'. This study argues that it is necessary to develop and activate a program that encourages women to maintain and recover a positive self-concept and self-esteem.

Conclusion: It is suggested that nursing care, policy, and social support related to infertility issues should be approached based on the positions, perspectives, and experiences of women who are undergoing infertility treatment in order to promote physical, mental, and socio-cultural health of them.

Key Words: Infertility, Women, Experience, Korea, Ethnography, Spradley's ethnographic analysis method

\section{INTRODUCTION}

Since August 2019, South Korea has become a country in which a woman never has one child in her life. The nation's total fertility rate is less than 1 . That is 0 people $(0.92)$. This is the lowest among OECD member countries. ${ }^{1,2}$ The number of infertile couples are rapidly increasing in Korean society for a variety of reasons, including increased marriage age, stress, environmental pollution, number of working couples, and increased ' 3 abandonments' tendency (i.e., younger generations tend to give up marriage, pregnancy and childbirth). ${ }^{1,3}$ And this is regarded as a major factor leading to lower fertility rates. ${ }^{4}$

Recently, infertility treatment in Korea has been highly technical and industrialized. On the other hand, as the low birth rate has treated as a national crisis, the Korean government is actively pursuing support programs for infertile couples. ${ }^{5,6}$ As the burden of expensive infertility treatment is reduced, infertility treatment procedures are increasing and becoming popular. ${ }^{6}$

Despite the rapidly changing values of the younger generation about families and children, married women are given a duty and role as the subject of childbirth in the socio-cultural context of Korea. ${ }^{7}$ And still, the idea, that the essence of femininity is 'birth and raising a child', is deeply embedded in the public mind. ${ }^{8}$

In Korean society, where child-centered values are more prevalent than couple-centered values, social relationships including families are being formed through the mediation of children. ${ }^{8}$ The birth of a child is a big socio-cultural event that strengthens the bonds between couples and family cohesion. ${ }^{9}$

If a married woman does not become pregnant within a year, the couple are judged infertile. ${ }^{2}$ Because pregnancy after marriage is expected as a natural outcome in the

\section{Corresponding Author:}

Dr. Seong-Heui Kim, Department of Nursing, Daejin University, South Korea; Email: nursingkim@daejin.ac.kr

ISSN: 2231-2196 (Print) ISSN: $0975-5241$ (Online)

Received: 10.06 .2020

Revised: 12.07 .2020

Accepted: 11.08 .2020

Published: 22.08 .2020 
socio-cultural context of Korea, infertile women are perceived as a 'abnormal', or 'incomplete' being. ${ }^{9} 10,11$ Infertility affects not only the female individual, but also the marital relationship, relationship with the in-laws, and social life, so the life of a woman diagnosed with infertility faces a total change and crisis. ${ }^{11,12}$ As soon as they are judged as infertile women, they are encouraged and pressured by the hospitals and their families to treat infertility. ${ }^{9,13}$ In the present situation, where infertility treatment is strongly supported at the national level, it has become a natural trend for infertile women to accept infertility treatment.

Breakthrough developments in reproductive technology are giving hope to infertile women. ${ }^{5}$ However, the current medical situation is concerned only with the success of pregnancy, and there is a lack of interest on women suffering from infertility treatment. ${ }^{14}$ It is reported that infertile women are undergoing a lot of physical, social, and psychological stress due to difficult tests, painful procedure, and long-term treatment. ${ }^{3,5,10,13}$ In particular, unlike couples who intentionally avoid pregnancy because they want to enjoy their own lives, the women, who choose infertility treatment, have a strong will and hope of the outcome of pregnancy and childbirth. ${ }^{15}$ That is why, infertile women have greater pain and conflict while undergoing repeated pregnancy failures in situations where pregnancy is not guaranteed.

With the combination of child-centered family culture in Korea, technological advances in infertility treatment, and the Korean government's childbirth support policy, number of the women receiving infertility treatment are rapidly increasing in Korean society. Domestically and internationally, researches on infertility have been mainly, pursued in the fields of medicine, psychology, religion, technology, and engineering. ${ }^{2,3,5,6,10,13-18}$ There is a lack of nursing research from an emic perspective on the experiences of women undergoing infertility treatment. A handful of nursing studies related to infertility are mostly survey studies using questionnaires, which are limited to in-depth understanding of the overall problems experienced by infertile women. ${ }^{3}$

Within the cultural background of Korean society, where social expectations or norms for traditional femininity still exist, it is very significant to understand in depth experiences of women undergoing infertility treatment from their point of view.

Culture is the belief, value, way of thinking, and lifestyle of a social group. And it is a frame of meaning that is shared among the members of the group. Since human reactions are formed in the culture to which they belong, the experiences of the subjects need to be understood in the socio-cultural context. ${ }^{8}$ Women undergoing infertility treatment form a subcultural group by sharing their experiences. In order to identify the cultural patterns shared by the cultural group and to understand them in depth, it is appropriate to apply an ethnographic research method. This leads to understanding of the cultural group by entering into their daily lives and describing their experiences from their point of view. ${ }^{19}$

Therefore, using this method, this study aims to explore and understand experiences shared by women undergoing infertility treatment in Korean sociocultural background, and finally provide the basic data for developing an effective nursing intervention for women to properly perform their gender role.

The main research question of this study is "What are the experiences of women undergoing infertility treatment in Korean society?"

\section{RESEARCH METHODOLOGY}

\section{Research design}

This is a qualitative research, using ethnographic research method in order to explain and understand the experiences of women who undergoing infertility treatment in the sociocultural background of Korea.

\section{Selecting research field and informant}

The research field of this study is the infertility treatment center of two women's hospital where this researcher is guiding students for nursing practice. It is regarded, therefore, as the best place in terms of accessibility of the study, feasibility for permission, and possibility of participation.

The informants are women undergoing infertility treatment at these hospitals. This researcher explained the aim and method of the study to the head nurse, who introduced appropriate informants, having over 10 years working experience at hospitals where this research was conducted. Informants were selected considering women's age, infertility period, existence of a job, religion, education level, and family type. Informants were selected in a manner of opportunistic, maximum variation and purposive sampling, as the data were being collected and analyzed, in on-going process.

The number of informants who reached the saturation level of data collection was 9. The general characteristics of the informants are as follows. Among the total 9 women undergoing infertility treatment, one was in their $20 \mathrm{~s}, 7$ were in $30 \mathrm{~s}$, and one was in 40s. Regarding their infertility type, 7 women were primary infertility and 2 were secondary infertility. The number of children in all informants was zero. Their infertility period ranged from 16 months to 6 years, with an average of 3 years and 1 month. In case of their marriage age, 2 were in their 20s, 6 in 30s, and 1 in 40s. As for their religious affiliation, 4 women were Christian, one was Buddhist, one was Catholic, and 3 had no religion. In terms of the cause 
of infertility, 5 women had female factor such as ovulation disorder, polycystic ovary syndrome, and endometriosis, and 4 were unknown. Regarding their occupation, 3 women indicated that they quit their jobs after starting infertility treatment, 2 were in regular jobs, one was on leave, and 3 were full-time housewives.

\section{Data collection and ethical consideration}

The data were collected during the period of August to December 2019 in the manner of ethnographic interviews and participant observation

The in-depth interview was conducted at the counseling room in infertility center. Interviews in the beginning were conducted in descriptive question to have outline of and search domain of the experience, and then gradually structural and contrast questions are followed in order to listen and observe what she has in her mind.

The main interview questions were: "How did infertility treatment begin?", "Could you tell me about your daily life during infertility treatment?", "What are difficult things during infertility treatment?", "Could you tell me your opinion about infertility treatment?"

Interview information was recorded on the researcher's cell phone under the consent of the informant, and after finishing the interview process, the recordings and notes were transcribed and confirmed authenticity by this researcher as soon as possible. Informants were interviewed twice or three times for 2 or 3 hours for each interview.

Participant observations were conducted during interviews of the informants. It is also observed at informants' daily spot-treatment unit, informant's home. Participation observation at the infertility center mainly consisted of attitudes and behaviors of infertile women, activities and attitudes of hospital officials, and hospital environment. In addition, facial expressions, speeches, and attitudes of husbands and families accompanying infertile women were also observed. educational materials provided by the infertility center, treatment schedule, and women's memo records were also used. The researcher visited an informant's home and participated in observations focusing on the informant's daily routine and the environment of the house. Particularly, participant observations in the informant's home was a meaningful time to confirm her will, beliefs, values, and practices for pregnancy.

The data were analyzed together with field notes which were immediately written after the participant-observations and information from informal on-site interviews. The data collection was done up to the saturation point, when new materials could no longer be found.

For ethical reasons, the data collection began after this researcher informed the informants about the spontaneity of research, the guarantee of the confidentiality of the research contents and utilization of the data for research purpose only, and then, accepted agreement of the informants to participate in this research. The informants were classified as numbers from 1 to 9 and marked them anonymously.

\section{Data analysis}

The data in this study were analyzed according to Spradley's ethnographic analysis method. This approach has a series of phases to discover and interpret the category, property, and cultural theme, from the various social situations through the process of domain analysis, taxonomic analysis, componential analysis, and theme analysis. ${ }^{19}$

In the first stage, the cultural domain of joys and sorrows of women undergoing infertility treatment was derived by identifying the overall outline based on extensive data collection and analysis ideas on the social situation of infertility treatment.

Second, in the taxonomic analysis phase, categorization was performed considering the semantic relationship between each other, based on data searched with focus on emotions, roles, activities, and relationships of women undergoing infertility treatment.

In the third stage, a componential analysis was conducted to find the properties of cultural categories.

Exploring the cultural theme and meanings conducted in the last step is the core of this research which confirms cognitive principle repeatedly appearing in a cultural group of women undergoing infertility treatment and finds the relationship between each part and the whole of culture.

Data analysis was done not by one-time process after completing all of the data collection, but by repeating process of disintegration, integration, comparison, modification, interpretation, and re- interpretation of participant observation and collected interview data. ${ }^{19}$ As a result, the patterns of experiences shared by women undergoing infertility treatment and its cultural meanings were found.

In order to secure the rigor of qualitative research, this study was conducted in accordance with four criteria proposed by Sandelowski, namely, credibility, fittingness, auditability, and confirmability. ${ }^{20}$ To ensure the validity and credibility of the study, the results of the data analysis were submitted for verification to the 2 informants, one nurse having over 10 years working experience at the infertility clinic of the hospital where this research was conducted, one nursing professors with ethnographic research experience.

\section{RESULTS}

Using ethnographic research method, as the result of exploring patterns of experiences on the joys and sorrows of 
women undergoing infertility treatment in Korean society and analyzing the cultural meanings, "getting rid of stigma of deficient women" was deduced as the cultural theme. This has meaning structure of 4 categories and 14 properties. The relationship between the cultural theme, categories, and properties is shown in below Table 1 .

The cultural theme was categorized as 'Turning into life of a loser', 'doing homework to be recognized for my existence', 'the ambivalence about infertility treatment', and 'positioning the value of being myself'. The identified properties in each category are as shown in Table 1.

Table 1: Relationships Between Cultural Theme, Categories, Properties

$\begin{aligned} & \text { Cultural Categories Properties } \\ & \text { Theme }\end{aligned}$

Turning into a Recommendations and preslife of a loser sures on normal family

Feeling shabby as an incomplete being

Doing home- Living up to the expectations work to be of family members

recognized for Trying to protect my position my existence as wife

Looking for an imposing but ordinary woman's life

Expectations and relying on medical technology

Getting rid of stigma of deficient women

The ambivalence about infertility treatment

All my daily routine focuses on pregnancy

Infertility treatment that provides comfort and hope

My body and mind becoming desolate

The discomfort for going against nature

Endless treatment like being in a swamp

Positioning the Expanding awareness of chil-

value of being dren and marriage myself
Accepting values centered on marital relationships

Strengthen my will to rechallenge for treatment

\section{Turning into a life of a loser}

This category is confirmed that 'recommendations and pressures on normal family' and 'feeling shabby as an incomplete being' as properties. It shows that informants are naturally forced to become pregnant after marriage, and they gradually experience a loss of self-esteem beyond the problem of

not getting pregnant in a social atmosphere that infertility women are not normal.

\section{Recommendations and pressures on normal family}

Most of the informants suffer from the pressure that they should have a baby as soon as they get married. And when they didn't get pregnant over time, they felt an atmosphere, both in their family and in society, passing on all the causes and responsibilities for infertility to them.

They said that Korea is a country where pregnancy is a mandatory course after marriage. Since the informants mainly married in the middle and late half of their $30 \mathrm{~s}$, both their parents and parents-in-law advised them not to use contraceptives after marriage, but try to get pregnant immediately. Even some parents encouraged them to have premarital pregnancy by saying that a child can be considered as the dowry since informants are too old. The same advice was given to informants who got married in their late 20s. This response from the parents of both families shows that the increasing number of infertile couples in Korean society is perceived as a social problem.

In Korean society, the birth of a child has traditionally been recognized to strengthen the bonds between couples and family cohesion. So, the common belief that married couples have to have a child to become a normal family is being naturally infused into married couples.

In addition, if a couple is not pregnant, the woman feels that the female, "I", has a whole responsibility for the problem of infertility, not only in the family relationship, but also in the diagnosis process. In other words, in contrast to the husband, who ends with just a semen analysis, during the various and difficult tests related to infertility performed on, the woman realizes that obligations of pregnancy and causes of infertility belong to her. From the process of being diagnosed with infertility, they strongly felt that pregnancy is thoroughly the woman's duty and the primary responsibility of the married women.

“...My parents said that everything will be alright if I get married, when I was a single. But, after married, it is now 'pregnancy', 'pregnancy'... Since both of us are getting old, I heard that even before marriage, I must give a birth quickly..."

"...A woman must have a healthy uterus so she can have children easily... Even my own mother is saying the same thing and worrying about...The reason why we couldn't get pregnant is unknown... therefore, naturally, they are saying that whether there is something wrong with me as a woman..."

“...Even my mother... says like this... Wanting to have children is human instinct. Moreover, women are more like that. 
It is difficult to maintain a family without children. Without children, husbands don't even want to come home."

“...It's completely different from my husband from the stage of examination. My husband just needs a semen test. If the test turns out no problem, and then, it is considered that all causes are up to me..."

Feeling shabby as an incomplete being: Informers have become infertile women naturally over a year after getting married as prescribed by modern medicine. They felt shameful, humble and desperate as if they were a loser in their lives, recognizing themselves as lacking being ${ }^{2}$. Because they thought they would not be able to do natural and normal process such as giving a birth, building a family and raising children. This shows that the childbirth is still an event that gives women a sense of achievement and meaning of life because the role of women is still considered to be the same as that of mothers in Korean society,

On the other hand, even doctors often emphasize that the success rate of pregnancy depends on the age of women rather than men. It is also pointed out by the people around them that the fundamental problem of infertility is always on the female side. In other words, there is a tendency to emphasize the cause of infertility to be a woman's problem. This unintentional social perception and common belief made women feel more guilty or miserable.

"... The doctor said that... You are unconditionally infertile if you are married for more than a year... So, I became a patient. I became an infertile woman... Naturally it became... I can't do anything else which other women can do... It a feeling like I'm an unable person short. This is also a kind of disability. Isn't it?"

“...Even if I see only a pregnant woman in an elevator, and even if I see a child at the playing ground of my apartment, I naturally shrank and became small... Because part of my life is still less fulfilled...."

"...cause is unknown, there is no cause of infertility, so the doctor said that I was the most likely to become infertile because I was older. It's harder to get pregnant if it's late... so let's start treatment as soon as possible... Everybody in my family are also naturally saying that it's infertility because I'm older. Because it is pinpointed as the most fundamental problem...."

“...Actually, even though my husband has some problems, I guess that my mother-in-law don't believe that... They think that women only need to be healthy. They have to ask my husband to drink less, quit smoking, and ask for something like this, but they say to me...I have to eat well to get pregnant... Even though I was diagnosed with no problems, I am wealthy."

\section{Doing homework to be recognized for my existence}

This category is confirmed that 'living up to the expectations of family members', 'trying to protect my position as wife', 'looking for an imposing but ordinary woman's life', 'expectations and relying on modern medical technology' as properties.

Living up to the expectations of family members: The informants came to accept infertility treatment in order to play the role assigned to them in the family, they had to take the responsibility for being pregnant and to adapt to infertility treatment for it. They said that they had chosen a way to be a good daughter-in-law, a good daughter and a good wife in their family. In the Korean family with strong Confucian succession, the role of women still tends to prioritize the function within the family that continues the family line through childbirth. Modern young women have a new perception of their children, but when they enter into family relations through marriage, they become subordinate to family relations regardless of their willingness to place them in marital relations.

This property shows that informants also have no choice but to be influenced by Korean traditional culture, which places more emphasis on parent-child relationships than marital relationships in family relationships.

“...Whenever I meet people after my marriage, they are greeting that 'Is there any good news yet?'... I thought that it's an inevitable thing in Korean society. I must have children in order to keep my marriage life... This kind of thinking is stuck in my mind unconsciously."

“...My own mother stood in a very hard situation in front of my parents-in-law...I also felt guilty for my mother with a burning heart... so I decided.”

“...I was in my 20 s when I married. According to today's standard, I was not too late age, so I decided to have a child after a few years... Even if my mother-in-law prompted me, I ignored that...But, when it was actually happened, I often had to concern and check my parents-in-law's feeling. I naturally feel like that 'Look at it.' 'Because you didn't pay attention to what I said..."

"...My mother-in-law said... The child is extending our life...My heart was stuffy..."

“...My parents-in-law told me... If you are stressed, it's harder to get pregnant... Don't come to our house on holidays or ancestral rites. Stay your home and rest only... They desperately want their grandchildren like this... at least I have to try."

Trying to pretect my positoin as wife: This property reveals the experiences of informants, choosing fertility treatment and going through a difficult course of treatment as 
a way to maintain their relationship and express their love towards their husbands, and fulfills the roles and responsibilities as a wife. Some women thought that meeting the expectations of their husband who desperately want children would qualify for the position of wife. They even thought that if they couldn't get pregnant, they would have to give up their position as a wife and be willing to divorce for their husband. Some informants have pledged their willingness to be pregnant with the wishes to give their husbands the pleasure of being a father. Even though their husbands did not express their expectations for children strongly, they would become more enthusiastic on treatment because of their gratitude and sorry for husbands who understood and cared about their situation. In common, they tried their best to do what they could in their position, while also expressing their potential anxiety about the future. They said that the extremely caring lives for them during an infertility treatment seemed to be like an ominous future collateral.

“...My husband really liked a child.... Once upon a time, just looking at them in the elevator and, smiling and said something. But... since I have become like this, he has never expressed like earlier in front of me... Pretend he is not interested even when he sees children... He greatly takes care of me. He also said to my parents-in-law that I have a problem ..."

"...I asked a pregnant woman how did it, and after took a note whatever she did, I and my husband tried to do everything"

“...I have eaten all kinds of food that's good for pregnancy. Not only do I go to a famous oriental medical clinic and make herbal supplements, but my mother takes the amulet and puts it in my pillowcase..."

Looking for an imposing but ordinary woman's life: This property is an experience in which infertile women continue treatment with the desire to become 'normal' and 'ordinary' women like other women, with feelings of shrinking about their own situation of infertility not only in family relationships and marital relationships but also in social relationships. They realized that, in Korean society, if they had no experience in childbirth and parenting, they would not be treated as mature adults even if they were married. In addition, in combination with work life and infertility treatment, they have always been subject to exceptions. In order to treat infertility, a considerate life was needed for them in both work and time. However, contrary to interest, women have often become more uncomfortable experiences with the special consideration of their family members or co-workers for themselves. So, they longed for the day when their infertility situation would end quickly and be treated as normal people like others.
"...Even when I have a dinner with other people at my company, they told me go to home quickly because it was hard for me... and these kinds of acts made me more atrophied."

“...I'm just an adult because I'm getting old... I'm still far from being an adult. I heard that I can be an adult after I gave a birth and raise the child... It seems like that I'm still immature and abnormal person."

"...Nowadays, people say that you have to give a birth to be a patriot. If I don't have a child yet, people say first that you do not contribute to the society."

Expectations and relying on medical technology: This property shows the experiences of infertile women dealing with great expectations and willingness to infertility treatment in an environment where the legitimacy and justification of the nation's massive support for infertility treatment have been obtained. In the Korean society, where low birth rate has become a national crisis, not only hospitals but also governments have directly or indirectly promoted the effectiveness and necessity of infertility treatment, and the state has strengthened economic and institutional support. From the diagnosis of infertility to the treatment, the rapid and remarkable development of technology and drugs gives hope to couples undergoing infertility. It has been reported that the identification of the causes of infertility and the development of various treatment methods have led to an increase in infertility specialized hospitals, and secondly, an increase in the number of couples diagnosed with infertility. It can be seen that this is consistent with the results of this study. On the other hand, in the past, Koreans had a rather passive response to treatment for infertility because the consciousness that pregnancy depends on the will of the sky (that is, God's will) was widespread among Koreans. However, as a result of the state's support and various promotional strategies for infertility treatments, it has changed the consciousness that infertility can only be overcome through active efforts and treatment. In particular, the change in consciousness of infertility treatment among the parents' generations acted as a factor forcing infertility treatment for infertile women. Indeed, one informant said that mother-in-law recommended infertility treatment by saying "Even people with disabilities are trying to give birth to children", after watching a TV program. These findings show some ambivalence of the impact of the development of the infertility treatment industry and the strong national support for infertility treatment on infertile women.

“...Before treatment, I mainly focused on folk remedies. But, after it did not work, then, my own mother went to see a fortune-teller. My mother was told that I do have a child according to my fortune. Since I'm getting older, I my not have a child who is in my fortune...It is only possible to have a child by going to hospital... I too started with that expectation." 
“...My mother-in-law saw a documentary about medical procedures for infertile couples on TV... After that, she said... it is a world where such disabled persons are too trying to give a birth..."

In this property, it can be seen that the experiences and pains of women undergoing infertility treatment did not receive attention from the surroundings and tended to be regarded as having to endure to have children.

\section{The ambivalence about infertility treatment}

This category is confirmed that 'all my daily routine focuses on pregnancy', 'infertility treatment that provides comfort and hope', 'my body and mind becoming desolate', 'the discomfort for going against nature', 'endless treatment like being in a swamp' as properties. Informants had comfort and hope in treating infertility, but on the other hand, they had a ambivalent feeling about the treatment by experiencing all the daily routines centered on pregnancy, the devastation of the body and mind, treatment procedures that are going against nature, and the characteristics of treatment that are not guaranteed but cannot be stopped.

All my daily routine focuses on pregnancy: This property demonstrates that, during infertility treatment, informants live a life for pregnancy only, lead a treatment-oriented life, and eventually have a skepticism and conflict about treatment as they feel that they are losing their lives. Informants were forced to leave or quit their jobs to treat infertility. Even women who remained at work often had to take vacations due to strict schedules and invasive procedures. As a result, they naturally became neglected in their work and became isolated in their relationship with their colleagues. In addition, because their entire daily life was obsessed with pregnancy, the choice of food and exercise was also related to this. Even watching TV or cell phone videos tended to be limited to topics related to pregnancy.

"... Since I started treatment, I had no choice but to quit my job. Reading faces at my office, I couldn't go to the hospital often. And the doctor too said that if I get stresses at work, the success rate is lower. So, if possible, it is better to quit my job..."

“...I don't know when I have to go to the hospital... So, I have to save my leaves for that day... Even if I want to have a rest, it is difficult..."

Infertility treatment that provides comfort and hope: This property shows that formants, who have been confused and despaired by the diagnosis of infertility, experience the comfort and stability of the mind with confidence and hope that they can become pregnant through fertility treatment.

“...Ah now, I can't be a mother anymore. If I couldn't have a child, I have to be divorced... I had a feeling of despair with such various kinds of thinking... But, because of the
IVF treatment, pregnancy is not impossible, the Doctor defined that 'it is only a condition of unfinished that has not yet been reached'... It was so comforting me. Instead, we must use assistive technology to become pregnant."

"...Unlike before, treatment is now also recommended by the government. Insurance benefits have also increased. So, I think it was much easier to decide to participate in the treatment."

It can be seen that the government's support for infertility treatment not only provided social legitimacy for infertility treatment, but also contributed to the popularization and positive awareness of infertility treatment. However, this social situation, contrary to the rate of interest, also resulted in driving infertile women to medical treatment rather than alternative methods. In other words, it spread the social atmosphere that women who were sentenced to infertility should naturally conceive and give birth to children through medical treatment.

My body and mind becoming desolate: This property reveals that infertile women experience psychological devastation due to repeated pregnancy failures as well as physical pain due to invasive procedures and side effects of treatment drugs. During the infertility treatment process, women were required to have strict schedules, invasive procedures, and intensive treatment. And, they suffered physical pain due to side effects and complications such as ovarian hyperstimulation syndrome, abdominal pain, general edema, and ascites caused by ovulation induction. In addition, women expressed fear of unproven side effects, worrying about the potential dangers of many drugs and invasive treatments administered to their bodies during infertility treatment. On the other hand, women experienced ambivalence, fear, and alienation until pregnancy is confirmed after in vitro fertilization and embryo transplantation. and then, when pregnancy failure is confirmed, they experienced loss, misery, depression, and loneliness. As the treatment period increased and the number of pregnancy failures increased, women suffered more severe physical, psychological, social and economic difficulties. In particular, the current medical situation focuses only on the success of pregnancy rather than considering the subject during the treatment process. So, it turns out that nursing care, concern, and consideration for the pain suffered by women being treated are neglected. It is necessary to define institutionally and manage at the national level so that the infertility treatment institutions provide clear explanations and data on the overall course of the procedure, possible situations, and side effects without exaggerating or distorting the results of infertility treatment.

“...There are some things that make my body difficult while taking a procedure... But most of all... how is my body going? What would I be later if I have got these kinds of drugs? I have such anxiety" 
"...What should I do if I fail this time?... I'm like an unstable examinee before the exam. And, when I failed again, then, my heart is really throbbing, I can't eat anything after that... Like that I again suffer for a few days...”

"...If menstruation is started, then, being depressed... Menstruation cannot be cursed like this...It has become so damned now. As this kind of cycle is continued, anxiety and depression become almost habitual..."

"...The woman, who was treated with me at this hospital, had ovarian hyperstimulation syndrome... To increase the rate of success, need to have a lot of ovulation...So, a lot of ovulation inducers are administrated to me... So my abdomen is distended and I can't breathe well...Actually, there are a lot of such women who are treated here... When I first started, there was not much explanation, and I was told only the successful cases."

The discomfort for going against nature: This property shows that informants continue to struggle due to the antipathy of artificial pregnancy and the unethical problems still being discussed during the process of infertility treatment. In particular, the fear of spoiling the natural flow of the body, the guilty feeling of neglecting life and invading the realm of God, and the antipathy of couple sex that were instrumentalized for pregnancy became a factor in strengthening the conflict over treatment.

“...Anyway... It's 'artificial'. The feeling of rejection on artificial things... Furthermore, about making a child artificially... Even if for going to bed, If I was told that it is most likely to get pregnant, if you make love such a time, then, I have to set the alarm clock and wake my husband up and then, have to demand...I felt a lot of sense of reluctance to do those things. That's the "human production plant" in the book The Brave New World. It is that kind of thing. Honestly speaking..."

“...Because I'm a Catholic, anyway, I continue to feel guilty of rejecting God... I keep thinking that there is something wrong with this. Many children are artificially fertilized, and if they become pregnant too much, then, ... Since can't give birth all of them, so getting rid of them again... A woman, was treated here with me, got pregnant with four, so, removed two and only two are left. So, making a life and kill it again."

In the course of treatment, most informants had a negative view on the behavior of hospitals that recommend and induce artificial insemination or in vitro procedures rather than a therapeutic approach to the cause of infertility. They were aware of the problem that hospitals focused on the way of using artificial assistive technologies, rather than enabling pregnancy in a 'normal' way, such as finding the cause of infertility and restoring their original function. Most doctors have emphasized the importance of testing for early detec- tion of infertility and starting treatment early, and dismissed attempting natural pregnancy for a long time as ignorant behavior.

"...My doctor said... the cause is ovulation disorder... But he didn't try to eliminate the cause by treating it fundamentally. It's not complete treatment... Only it's a temporary method... From the perspective of the hospital... because these methods make them more money than natural pregnancies."

“...Feeling like my body's being manipulated by someone...I feel like I can't control my body... I hate it so much."

Endless treatment like being in a swamp: This property shows the experience of being skeptical and anxious as informants become weary of days of undetermined treatment that endure with a vague dream of becoming a mother. They said that the longer the period of infertility treatment, the more loss of control over childbirth and the more difficult it was to decide when to stop treatment. The treatment process requires a long time and economic burden. In reality, Infertility treatment does not necessarily guarantee pregnancy. However, once they started treatment, it was not easy to stop because it gave them vague hope that someday in the process, they would succeed. On the other hand, they worried about the unstable future as their careers were continuously cut off and the economic burden increased.

"... Ovulation is good enough and there are no problems... But, because it keeps failing... I have to stop it after doing this time... I thought this is the last time...But, when I hear that somebody get pregnant at this time, I have a hope that I too will have one day... Since I could not give it up, getting crazy. It's like a homework that never ends."

"... The words that the doctor said continued in my head... The more try, the higher the success rate... Some people say they have a child after five years of treatment... there are people who are pregnant at 44 years of age... I have vague expectations that one day I will succeed, and on the other hand, I am worried that I will have a bigger regret next time...."

“...I quit my job for this treatment and it's been 3 years already. It's hard to go back and work again. It's a computer processing job..."

“...Like this, I can't do my job forever, and if I can't have a child, I have to work... Extremely, I have to consider even divorce and live... I can't do anything now and It is not easy to find a job... My future is insecure both in my family as well as in the society."

\section{Positioning the value of being myself}

This category is confirmed that 'expanding awareness of children and marriage', 'accepting values centered on marital relationships', 'strengthen my will to re-challenge for treatment' as properties. 
Expanding awareness of children and marriage: This property means that, after several attempts and failures of pregnancy, women take a step back from their obsession with pregnancy, taking the time to look back on themselves who had been cling to treatment. In other words, this shows that they are willing to wait for pregnancy with a relaxed mind or change their perception of children and marriage. Informant considered alternatives such as relying on religion, concerns about adoption, or even divorce to choose a life free from the bridle of marriage, beyond the way they have so far relied solely on infertility treatment. Some informants have said that adoption is not an easily acceptable alternative in Korean society, where there is a strong negative mood for adopting children of others. Some prepared themselves to leave their positions and end their ties in order to be free from this suffering when there were no more alternatives.

"I was so obsessed with infertility treatment... I was so nervous...So, maybe, I can't get pregnant... In some cases, after stopping treatment, somebody became pregnant naturally... I started this treatment right after I got married and a year later... In a way, the period of attempting natural pregnancy is shorter. Going to the church with a little peace of mind, and if I'm doing my daily life, maybe there's something better..."

"Now, celebrities adopt publicly... and people see them with good eyes. I don't have to think negatively about adoption... I'm opening my mind little by little..."

"...I'm thinking a lot about divorce these days. I thought that If I live like this, I would rather live alone. I wonder how good this position is, and should I live like this uncomfortable for my lifetime. It is better to hear I am a divorcee and live rather than to hear I am infertile and disabled..."

Accepting values centered on marital relationships: This property means that infertile women are newly prepared to establish a new couple-centered value rather than a childcentered one and to cherish life with their husband. Women realized that infertility treatments made their relationship with their husbands worse, and thought that the lives of existing couples were more precious and worth keeping than non-existent children.

"...When I just think of a child, I thought it is not easy to continue our marital relationship anymore. This is not a fun relationship, but a mandatory, like doing homework mechanically...The relationship for just pregnancy is miserable. This is not a living... Sincerely... If we want to continue to live as a couple, we have to abandon our obsession a little with a child."

“...For a long time, due to having treatment, I could not travel well with my husband. Now the lives of both of us are also important... Because of a child who doesn't exist, and our life has been so miserable for a long time after married... Now, I think of living our lives..."
Strengthen my will to re-challenge for treatment: This property demonstrates the characteristics of infertile women who endlessly challenge and do their best with the determination to maintain a traditional attitude of emphasizing children and to actively and consistently maintain treatment for pregnancy. Informants showed their willingness to try to get pregnant by taking other measures, such as moving to hospitals known to have a high pregnancy success rate or strengthening their bodies.

"...I hope that someday I will have a normal family. Due to the sincerity of my family member and my mother... I can't let my efforts, that I've tried so far, become a bubble."

The category of 'positioning the value of being myself' shows that women, who experience repeated failures of pregnancy, are not sticking to vain expectations of infertility treatment, but rather growing through the process of expanding their perceptions of children, embracing marital values, and solidifying their will to new challenges.

\section{DISCUSSION}

The number of women undergoing infertility treatment is rapidly increasing due to mainly three factors, the childcentered family culture, technological advances in infertility treatment, and the Korean government's childbirth support policy. This study used an ethnographic research method to deeply explore experiences of women undergoing infertility treatment in the Korean socio-cultural context, and to understand the patterns and meaning of the experiences of the women.

This study found that a common experience of women undergoing infertility treatment in Korean society was "getting rid of stigma of deficient women." This means the cognitive principle repeatedly appeared in the culture of women group receiving infertility treatment. Through this study, it was confirmed the perception, that the main role and responsibility of married women is childbirth and parenting, is still deeply rooted in modern Korean society. So that, infertile women experienced the feelings of being a loser in life, recognizing themselves as lacking being, 'not normal'.

In addition, it showed cultural patterns that they relied on fertility treatment while looking forward to the lives of ordinary women and expecting to be recognized for their existence in the family and society, but eventually they could not have normal lives.

It was revealed that infertile women had comfort and hope in treating infertility, but on the other hand, they had an ambivalent feeling about the treatment by experiencing all the daily routines centered on pregnancy, the devastation of the body and mind, treatment procedures that are going against nature, and the characteristics of treatment that are not guar- 
anteed but cannot be stopped.

While experiencing repeated failures of pregnancy, women have the opportunity to expand their perception of children, or to accept marital-centered values rather than clinging to excessive expectations of infertility treatment. Otherwise, they redefined their will for new challenges while observing the success of pregnancy around them. Eventually, it represented cultural patterns that women are experiencing the mature process of establishing their own values.

It is significant that this study reveals the experiences of women who have been thoroughly excluded as a subject of treatment in the infertility industry, which has been strongly supported as part of the nation's childbirth policy.

Based on the results of this study that highlights the experiences of women undergoing infertility treatment in the context of Korean sociocultural context, it is required that approaches to promote their physical, psychological and sociocultural health be made in various fields.

\section{CONCLUSION}

In terms of nursing practice, for the subjects before taking the treatment, it is necessary to provide nursing education on the process, risks, side effects, and outcomes of infertility treatment. Since infertile women begin the treatment with excessive expectation that they will be able to become pregnant only if they take it, they struggle with the reality of repeated pregnancy failures and, physical and psychological pain. Therefore, it is suggested to establish institutional process systematically, so that they can adequately cope with the treatment process by having sufficient information on the effects of infertility treatment. In addition, there are few support programs in their families and communities that help women get psychological support and stability during infertility treatment. In particular, development and activation of programs that encourage women to rebuild positive self-concepts and self-esteem is urgently needed.

Currently, issues of infertility in Korea mainly focus on the women or couples as their personal matters. Therefore, it is necessary to concern infertility matters as an important social issue and responsibility.

At the policy level, the government should make various institutional supports and efforts to encourage couples to recognize themselves that they need children, and to make decisions to build a healthy family, rather than emphasizing and supporting infertility treatment as a way to increase the fertility rate. This is because childbirth is associated with various social factors such as employment, housing issues, parenting and education, and matter of work and life balance.
Since this study was conducted on nine women undergoing infertility treatment in two hospitals, it is limited to generalize the results of the study, even if the data collection reached saturation level.

\section{ACKNOWLEDGEMENT}

Authors acknowledge the immense help received from the scholars whose articles are cited and included in references of this manuscript. The authors are also grateful to authors / editors / publishers of all those articles, journals and books from where the literature for this article has been reviewed and discussed.

\section{Conflict of Interest: Nil}

Funding Source: Nil

\section{REFERENCES}

1. Statistics Korea. Infertility Experience of Married Women (15 49 years) and Husbands. Statistic Korea 2019.

2. Lee YS, Choi SM, Kwon JH. Psychosocial Predictors of Infertile Women's Distress. Journal of the Korean Society of Maternal and Child Health. 2019 ;23(2):136-46.

3. Kim M, Nam H, Youn M. Infertility stress, depression, and resilience in women with infertility treatments. Journal of Korean Public Health Nursing. 2016;30(1):93-104.

4. Seong-Heui Kim. An Ethnography on Joys and Sorrows of Women Undergoing Infertility Treatment in Korea. International Journal of Social Welfare Promotion and Management. 2020;7(2):25-32.

5. Peters K, Jackson D, Rudge T. Failures of reproduction: problematising 'success' in assisted reproductive technology. Nursing Inquiry. 2007;14(2):125-31.

6. Hwang N, Jang I. Factors influencing the depression level of couples participating in the national supporting program for infertile couples. Journal of Korean Academy of Community Health Nursing. 2015 ;26(3):179-89.

7. Eunsil Kim. An Analysis of Research Trends in the Use and Abuse of Alcohol by Adolescents: Papers Published in Korean Academic Journals. International Journal of Advanced Nursing Education and Research. 2018; 3(2):19-24.

8. Kim SH, Yoo EK. Ethnographic research on adjustment of mothers caring for their cancer children in Korea. Korean Journal of Women Health Nursing. 2015;21(3):216-31.

9. Ulrich M, Weatherall A. Motherhood and infertility: Viewing motherhood through the lens of infertility. Feminism \& Psychology. 2000 Aug;10(3):323-36.

10. Gourounti K, Anagnostopoulos F, Vaslamatzis G. Psychosocial predictors of infertility related stress: A review. Current Women's Health Reviews. 2010 ;6(4):318-31.

11. SH Kim. A Study of Unmarried Women's Experiences on Illegal Abortion in South Korea. Asia Life Sciences Supplement. 2019;19(2):143-154.

12. Jin-Hee Kim, Hye-Jeong Choi, Hyeong-Kwan Kim. The Effect of Obesity on Adolescent Development: Relationship between Self-Efficacy, Parent-Child Relationship, Peerlessness, and SelfEsteem. International Journal on Consulting Psychology for Patients. 2018;2(2):69-76.. 
13. Pasch LA, Sullivan KT. Stress and coping in couples facing infertility. Current opinion in psychology. 2017 ;13:131-5.

14. Waldby C, Cooper M. The biopolitics of reproduction: PostFordist biotechnology and women's clinical labour. Australian Feminist Studies. 2008;23(55):57-73.

15. JWM Aart, V Empel, J Boivin, WL Nelen, JAM Kremer, C. M. Verhaak. Relationship between Quality of Life and Distress in Infertility; a Validation Study of the Dutch FertiQoL. Human Reproduction. 2011;26(5):11124-1118.

16. Yeon-Gyung Bak. The Effect of Anger Control Education on Women's Breast Cancer Patients. International Journal on Consulting Psychology for Patients. 2018;2(2):7-12.
17. Boivin J, Takefman J, Braverman A. The fertility quality of life (FertiQoL) tool: development and general psychometric properties. Human Reproduction. 2011;26(8):2084-91.

18. De Lacey S. IVF as lottery or investment: contesting metaphors in discourses of infertility. Nursing Inquiry. 2002;9(1):43-51.

19. Link GJ. Cooperative Signaling Behavior: Signals for Open Source Project Health (Doctoral dissertation, University of Nebraska at Omaha).

20. Sandelowski M. The problem of rigor in qualitative research. Advances in nursing science. 1986:27-37. 